\title{
Pain level between clear aligners and fixed appliances: a systematic review
}

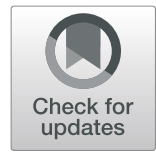

\author{
Paula Coutinho Cardoso ${ }^{1}$, Daybelis Gonzalez Espinosa', Paulo Mecenas ${ }^{1}$, Carlos Flores-Mir ${ }^{2}$ and \\ David Normando ${ }^{3^{*}}$ (i)
}

\begin{abstract}
Objectives: To assess if there is any difference in pain levels between orthodontic treatment with clear aligners or fixed appliances.

Materials and methods: An electronic search was completed in PubMed, The Cochrane Database, Web of Science, Scopus, Lilacs, Google Scholar, Clinical Trials, and OpenGrey databases without any restrictions until February 2019. All comparative study types contrasting pain levels between clear aligners and fixed appliances were included. The risk of bias (RoB) was assessed using the Newcastle-Ottawa Scale, ROBINS-I-Tool, or ROB 2.0 according to the study design. The level of evidence was assessed through the GRADE tool.

Results: After removal of duplicates, exclusion by title and abstract, and reading the full text, only seven articles were included. Five were prospective non-randomized clinical trials (CCT), one was a cross-sectional study, and one was a randomized clinical trial (RCT). Two studies presented a high RoB, three a moderate RoB, and two a low RoB (including the RCT). A meta-analysis was not performed because of clinical, statistical, and methodological heterogeneity. Most of the studies found that pain levels in patients treated with Invisalign were lower than those treated with conventional fixed appliances during the first days of treatment. Differences disappeared thereafter. No evidence was identified for other brands of clear aligners.

Conclusions: Based on a moderate level of certainty, orthodontic patients treated with Invisalign appear to feel lower levels of pain than those treated with fixed appliances during the first few days of treatment. Thereafter (up to 3 months), differences were not noted. Malocclusion complexity level among included studies was mild. Pain is one of many considerations and predictability and technical outcome are more important, mainly considering that the difference does not seem to occur after the first months of the orthodontic treatment.
\end{abstract}

Keywords: Orthodontic appliances, Pain, Invisalign, Malocclusion

\section{Introduction}

Pain is a subjective response and presents a large number of individual variations under the same trigger conditions. It depends on several factors such as age, sex, individual pain threshold, emotional state, stress, amount of applied force, cultural differences, and previous experiences of pain $[1,2]$. Pain complaints are a common feature during orthodontic treatment [3] directly influencing patient's satisfaction [4]. It is one of the main reasons for orthodontic treatment discontinuation [5].

\footnotetext{
* Correspondence: davidnormando@hotmail.com

${ }^{3}$ Faculty of Dentistry, Federal University of Pará (UFPA), Rua Augusto Correa

01, Belém, Pará 66075-110, Brazil

Full list of author information is available at the end of the article
}

It is well known that during orthodontic treatment with fixed appliances, it is common to feel pain and discomfort [6], reaching its peak $24 \mathrm{~h}$ after arch insertion, and being almost imperceptible 7 days after $[7,8]$. However, the type of the appliances may have an influence on the pain and discomfort reported by the patients due to the type of force applied. Removable appliances produce intermittent forces, which allow the tissues to reorganize before compressive forces are reapplied [9]. Regarding studies that have evaluated pain levels with clear aligners compared to fixed appliances, some studies have found positive $[2,10,11]$ or negativ e[12] results related to clear aligners. 
When comparing quality of life (QoL) between patients treated with fixed appliances and Invisalign (Align Technology, San Jose, CA), it was observed that both presented similar QoL results, except under the category of eating and chewing where the aligner group showed better results [13].

Systematic reviews have evaluated the efficiency of orthodontic treatment with clear aligners and they suggested that the outcomes are not as accurate as those with fixed appliances [14-16]. On the other hand, treatment done with clear aligners present some advantages such as overall decreased treatment and chair time in patients with mild to moderate malocclusions [17]. Besides that, studies have shown that gingival health tends to be better, based on the periodontal health index, in patients treated with clear aligners $[18,19]$.

There are controversial findings regarding pain level during orthodontic treatment with fixed appliances versus clear aligners. Thus, the aim of this systematic review was to evaluate the level of pain during orthodontic treatment in patients treated with clear aligners compared with patients treated with fixed appliances.

\section{Material and methods}

\section{Protocol and registration}

The present systematic review was registered in the PROSPERO database (http://www.crd.york.ac.uk/PROSPERO, PROTOCOL: CRD42019131359) and was done according to the Preferred Reporting Items for Systematic Review and Meta-Analysis (PRISMA) guidelines (www. prisma-statment.org).

\section{Eligibility criteria}

The following criteria were used in the selection of the articles:

1. Study design: Randomized or non-randomized controlled clinical trials and cross-sectional studies.

2. Population: Adult patients during orthodontic treatment.

3. Intervention: Patients treated with clear aligners.

4. Comparison: Patients treated with conventional fixed appliances.

5. Outcome: Pain level.

6. Exclusion criteria: Laboratory studies, clinical trials, case reports, literature reviews, and studies done with patients with syndromes and/or craniofacial deformities were excluded from the research.

Information sources, search strategy, and study selection There were no restrictions on languages and dates of publication. The search was completed by two authors (P.C.C and D.G.E.) until February 2019. The search was performed in the following databases: Cochrane,
PubMed, Scopus, Google Scholar, Lilacs, Web of Science, Clinical Trials, and OpenGrey. Specific search strategies per database are shown in Appendix 1. A hand search was also performed.

The included articles were exported to a bibliography reference manager (Mendeley, version 1.19.4 Elsevier). In case of disagreement, a third evaluator (D.N) opinion was consulted.

\section{Data items and collection}

The data collection in duplicate was carried out according to the following criteria: type of study, sample size, intervention, assessment of pain, time of evaluation, sequence of the archwires and aligners, pain outcomes, overall outcomes, analgesic consumption and author's conclusion (Table 1).

\section{RoB/quality assessment in individual studies}

For the cross-sectional study, the Newcastle-Ottawa Scale adapted to cross-sectional studies was used [21]. The evaluation was done by counting stars acquired in each category (Table 2).

For the evaluation of RoB for the non-randomized clinical trials, the ROBINS-I-tool [22] was used. The evaluated criteria were divided into pre-intervention, intervention, and post-intervention categories. The RoB was individually analyzed for each study and classified as low, moderate, serious, critical, and no information (Table 3).

For the randomized clinical trial, the RoB was performed using Cochrane Collaboration RoB 2.0 tool [23], analyzing six domains: random sequence generation, allocation concealment, blinding of patients and personnel, blinding of outcome assessor, incomplete outcome data, and selective outcome reporting (Table 4).

\section{Summary measures}

Clinical heterogeneity was measured assessing the treatment protocol, according to the archwire sequences and use of the aligners, times of evaluation of pain levels, use of analgesics during orthodontic treatment, different prescriptions of the fixed appliances, and other outcomes such as soft tissue irritation and eating disorders. The assessment of pain levels was evaluated through a visual analog scale (VAS).

For continuous outcomes, descriptive statistics, such as mean differences and standard deviations, were used to summarize the data from the included studies.

\section{RoB/quality assessment among studies}

The quality of evidence of the included studies was made according to The Grading of Recommendations Assessment, Development and Evaluation (GRADEpro 


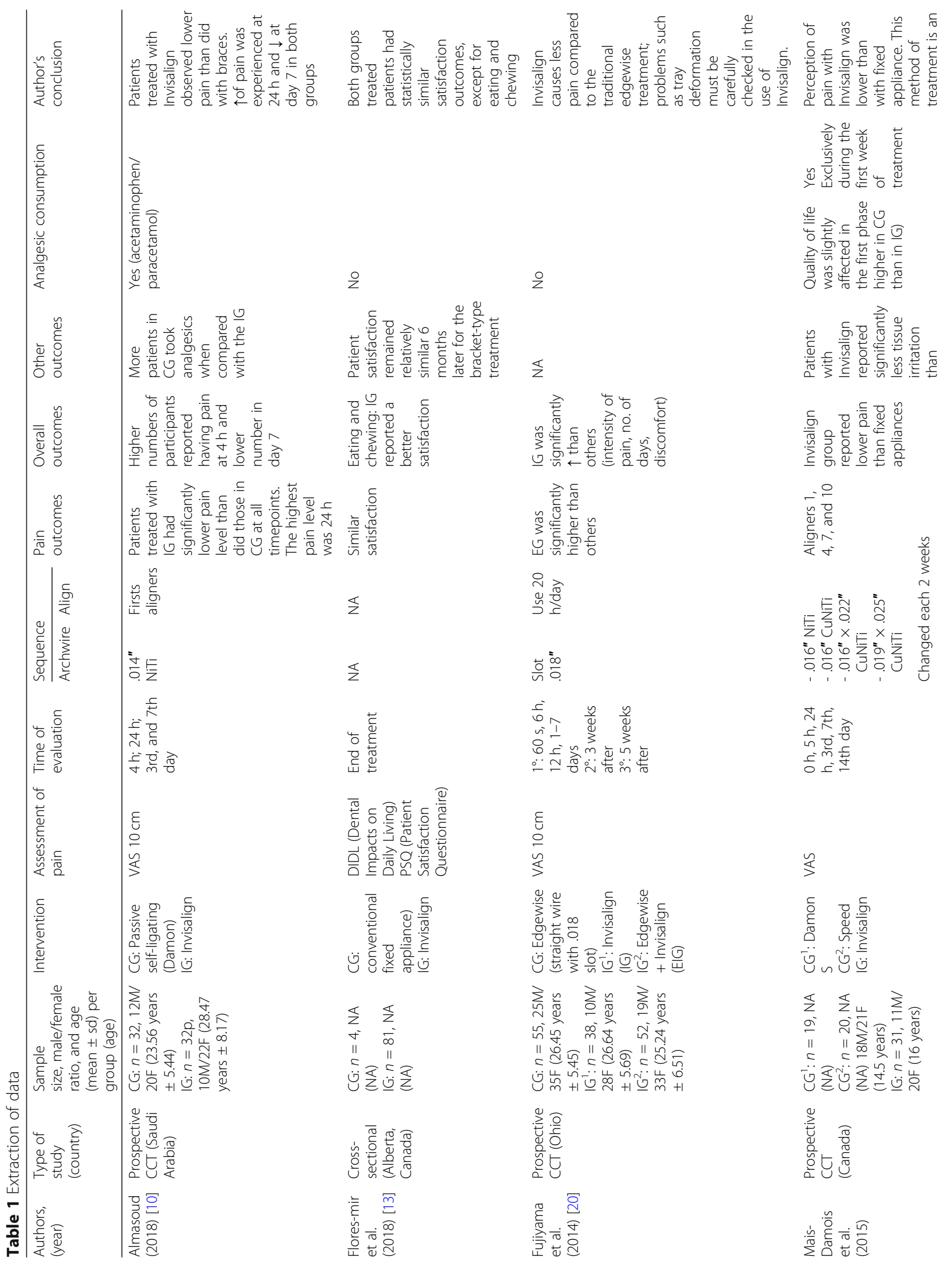




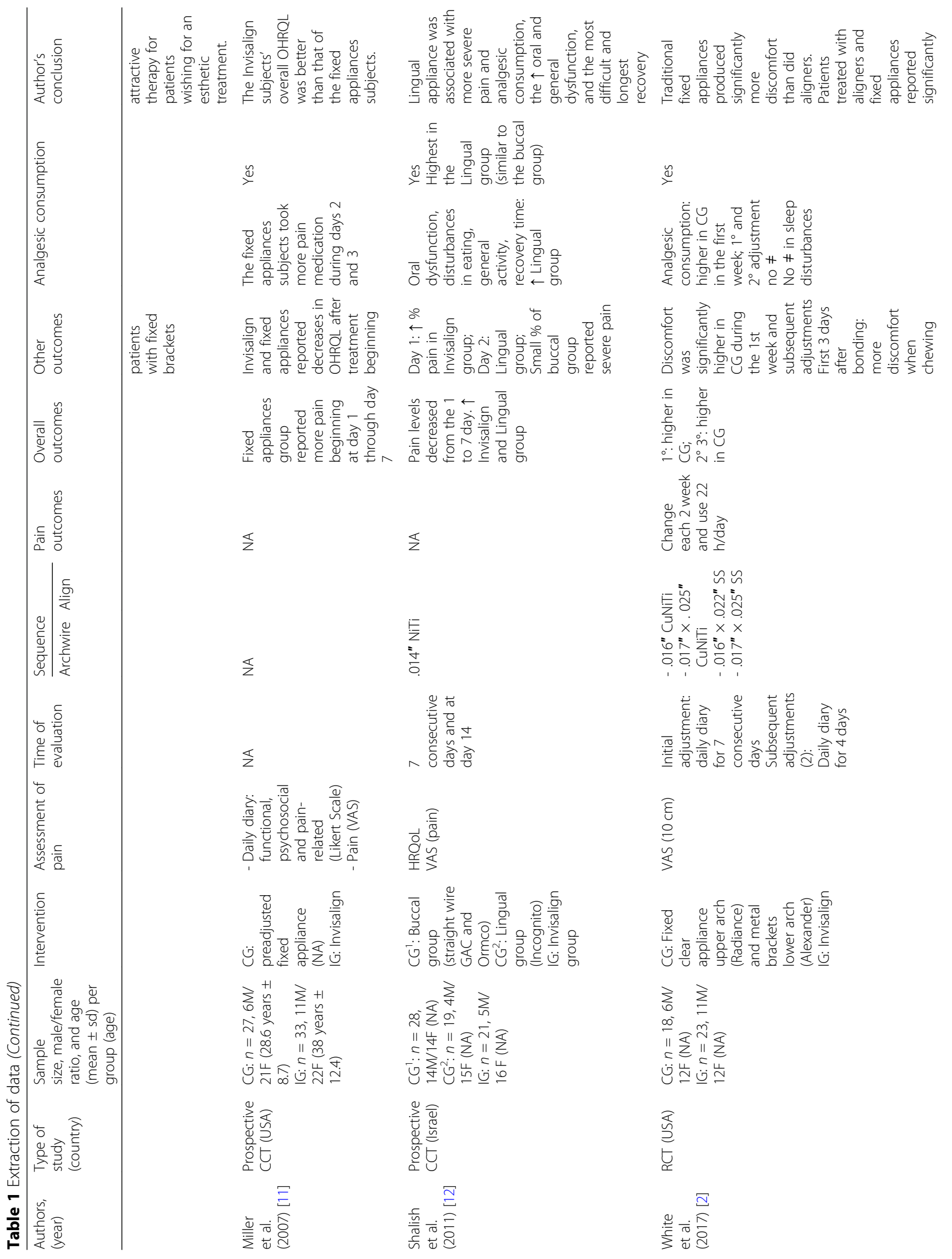


Cardoso et al. Progress in Orthodontics $\quad$ (2020) 21:3

Page 5 of 17

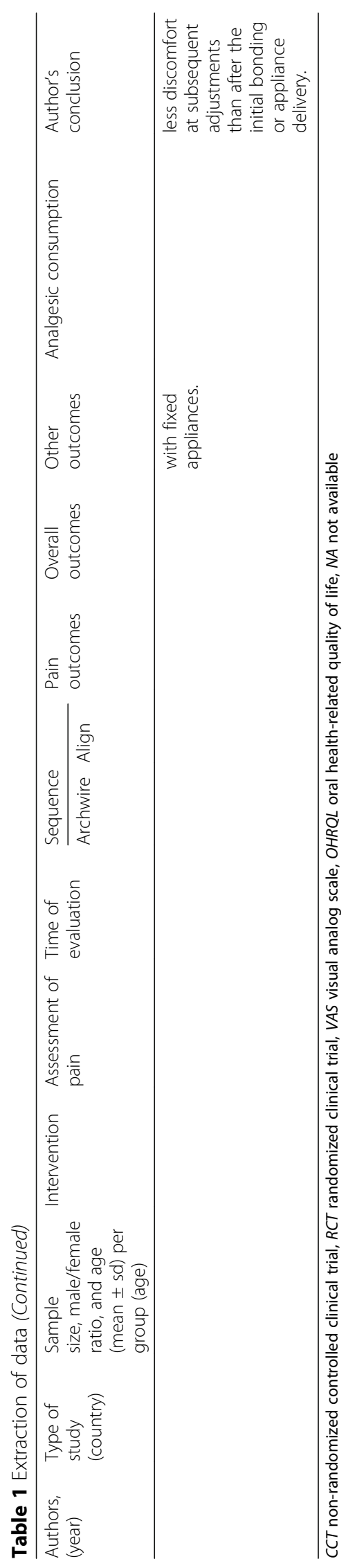


Table 2 Risk of bias of the studies, according to the Newcastle-Ottawa Scale adapted for cross-sectional studies

\begin{tabular}{lllll}
\hline & Selection (maximum 5 stars) & Comparability (maximum 2 stars) & Outcome (maximum 5 stars) & Total score (maximum 10) \\
\hline Flores-Mir et al. 2018 [13] & 4 & 1 & 3 & 8 \\
\hline
\end{tabular}

Newcastle-Ottawa Quality Assessment Scale (adapted for cross sectional studies)

Selection (maximum 5 stars):

1. Representativeness of the sample:

a)Truly representative of the average in the target population. * (all subjects or random sampling)

b)Somewhat representative of the average in the target population. * (nonrandom sampling)

c) Selected group of users.

d)No description of the sampling strategy.

2. Sample size:

a) Justified and satisfactory. *

b) Not justified.

3. Non-respondents:

a) Comparability between respondents and non-respondents characteristics is established, and the response rate is satisfactory. *

b) The response rate is unsatisfactory, or the comparability between respondents and non-respondents is unsatisfactory.

c) No description of the response rate or the characteristics of the responders and the non-responders.

4. Ascertainment of the exposure (risk factor):

a) Validated measurement tool. **

b) Non-validated measurement tool, but the tool is available or described.*

c) No description of the measurement tool.

Comparability (maximum 2 stars):

1. The subjects in different outcome groups are comparable, based on the study design or analysis. Confounding factors are controlled.

a) The study controls for the most important factor (select one). *

b) The study control for any additional factor. *

Outcome (maximum 3 stars):

1. Assessment of the outcome:

a) Independent blind assessment. **

b) Record linkage. **

c) Self-report. *

d) No description.

2. Statistical test:

a) The statistical test used to analyze the data is clearly described and appropriate, and the measurement of the association is presented, including confidence intervals and the probability level ( $p$ value). *

b) The statistical test is not appropriate, not described or incomplete

Guideline Development Tool, available online at gradepro.org) [24].

Table 3 Risk of bias of included articles

\begin{tabular}{ll}
\hline Domain bias & Description \\
\hline $\begin{array}{l}\text { Pre-intervention } \\
\text { Bias due to confounding }\end{array}$ & $\begin{array}{l}\text { Assessment of baseline in of certain number of participants by age and malocclusion description } \\
\text { Assessment of the method of pain evaluation } \\
\text { Assessment of time of evaluation }\end{array}$ \\
$\begin{array}{l}\text { Bias in selection of participants into } \\
\text { the study }\end{array}$ & $\begin{array}{l}\text { Assessment of participants eligibility criteria } \\
\text { Evaluation of eligible participants exclusion or difference between the follow-up period }\end{array}$ \\
$\begin{array}{ll}\text { Intervention } \\
\text { Bias in classification of interventions }\end{array}$ & $\begin{array}{l}\text { Assessment of the intervention status-use of the aligner was not properly described (change of the } \\
\text { aligner) } \\
\text { Use of additional orthodontic methods to correct malocclusion (ex: Ml, elastic) } \\
\text { Use of analgesic }\end{array}$
\end{tabular}

Post-intervention

Bias due to deviations from intended interventions

Bias due to missing data

Bias in measurement of the outcomes

Bias in selection of the reported results
Evaluation of the systematic differences between the intervention (group that used the aligner) and the comparison group when there is no information about the evaluation of the pain Use of analgesic for pain relief during orthodontic treatment

In the event of loss of follow-up, incomplete collection of data and exclusion of participants from the analysis

When assessments of pain perception were not reported or were measured with error When not all the measures established in the different treatment times are presented When the use of analgesic is mentioned or not

Selective report of results when the effect of all measurements of results has not been fully reported 
Table 4 Risk of bias of included studies

\begin{tabular}{lllllll}
\hline \multirow{2}{*}{ Study } & Risk of bias & & & & \\
\cline { 2 - 6 } & $\begin{array}{l}\text { Random sequence } \\
\text { generation }\end{array}$ & $\begin{array}{l}\text { Allocation } \\
\text { concealment }\end{array}$ & $\begin{array}{l}\text { Blinding of patients, } \\
\text { personnel }\end{array}$ & $\begin{array}{l}\text { Blinding of outcome } \\
\text { assessor }\end{array}$ & $\begin{array}{l}\text { Incomplete } \\
\text { outcome data }\end{array}$ & $\begin{array}{l}\text { Selective outcome } \\
\text { reporting }\end{array}$ \\
\hline White et al. (2017) [2] & Low & Low & Low & Low & Low & Low \\
\hline
\end{tabular}

\section{Synthesis of results}

A meta-analysis was not justifiable because of the large amount of clinical, statistical, and methodological heterogeneity.

\section{Results}

\section{Study selection and characteristics}

A total of 1773 studies were identified in the following databases: PubMed (663), Cochrane (124), Web of Science (68), Scopus (13), Lilacs (2), Google Scholar (895) Clinical Trials (5), and OpenGrey (3). A manual search was also undertaken but no articles were found. The identified articles were exported to the Mendeley Desktop (version 1.19.4) reference manager to remove duplicates, and a total of 1625 articles remained after the duplicates were removed. A flow diagram of the process of identification, inclusion, and exclusion of studies is presented in Fig. 1.

The exclusion of articles by title and abstract was done by two evaluators (PC and DG), and in the end, 29 studies were selected to be evaluated by full text. Of these, 22 were excluded because 2 were a literature review, 14 did not have a control or an intervention group, 3 failed to evaluate pain, and 3 were not related to the objectives of this systematic review (Table 5).

A total of seven studies were finally included. Five were prospective non-randomized clinical studies [10$12,20,25]$, one was a cross-sectional study [13], and one was a randomized clinical trial [2]. The mean age of the control group (group with fixed appliances) ranged between 23.56 [10] and 28.6 [11], but four studies [2, 12, $13,25]$ did not report this information. A homogeneity was observed due the type of aligner used in all studies (Invisalign aligner); however, different types of fixed appliances were used as a control group, such as Edgewise [20], Damon Q (Ormco, Orange, CA) [10, 25] (Ormco, Glendora, CA), Speed [25] (Strite Industries Ltd., Ontario, Canada), Radiance (American Orthodontics, Sheboygan, WI) in the maxillary arch, and Alexander (American Orthodontics, Sheboygan, WI) in the mandibular arch [2]. One study just reported a use of a straight-wire appliance from GAC or Ormco [12], and two studies did not report any information [11, 13].

In five studies $[2,10,20,25,26]$, a VAS was used as a method for evaluating pain level, one study used a questionnaire at the end of treatment [13], and two studies used both methods $[11,12]$.
When evaluating follow-up time, six studies [2, 10-12, $20,25]$ reported daily evaluations during 1 week until 3 months of follow-up, and only one study evaluated pain level at the end of treatment [13].

\section{RoB within studies}

The Newcastle-Ottawa scale for cross-sectional studies was applied for one study [13] and was classified with a good quality of evidence. A lower grade was applied for the selection domain due to the representativeness of the sample that was ranked as selected groups of users.

The ROBINS-I-Tool (Risk of Bias in Non-randomized Studies-of Interventions) was used in five studies [1012, 20, 25] (Table 1). Reasons related to increased RoB included confusing information (description of the malocclusion, method of pain evaluation, and follow-up time). Only one [10] study presented a low RoB and four $[11,12,20,25]$ showed a moderate RoB (Table 6). The major reason for this grading was related to the use of analgesics and this information was not properly reported (use or not). One study [11] was classified as high RoB in the intervention domain due to bias in classification of interventions, which included the assessment of the intervention status, use of additional orthodontic methods to correct, and use of analgesic.

For the randomized clinical trial [2], RoB was evaluated according to the Cochrane Collaboration RoB 2.0 tool, which presented a low RoB in all domains: random sequence generation, allocation concealment, blinding of patients and personnel, blinding of outcome assessor, incomplete outcome data, and selective outcome reporting (Table 3).

\section{Summary of individual studies' results}

Among all included studies [2, 10-13, 20, 25], pain scores were obtained $24 \mathrm{~h}$ after the beginning of treatment, and four included articles reported higher pain levels for fixed appliances during this period. However, only one investigation [10] found a statistically significant difference. Two others studies $[2,11]$ only reported a significant difference on day 3 and on day 4 . Both studies reported that pain levels were higher in the group treated with fixed appliances. During days 5-7, only one study [2] observed a significantly higher level of pain in the patients with fixed appliances, but the highest level of pain was on the third day. Two studies [12, 25 ] evaluated pain on day 14 and reported no significant 


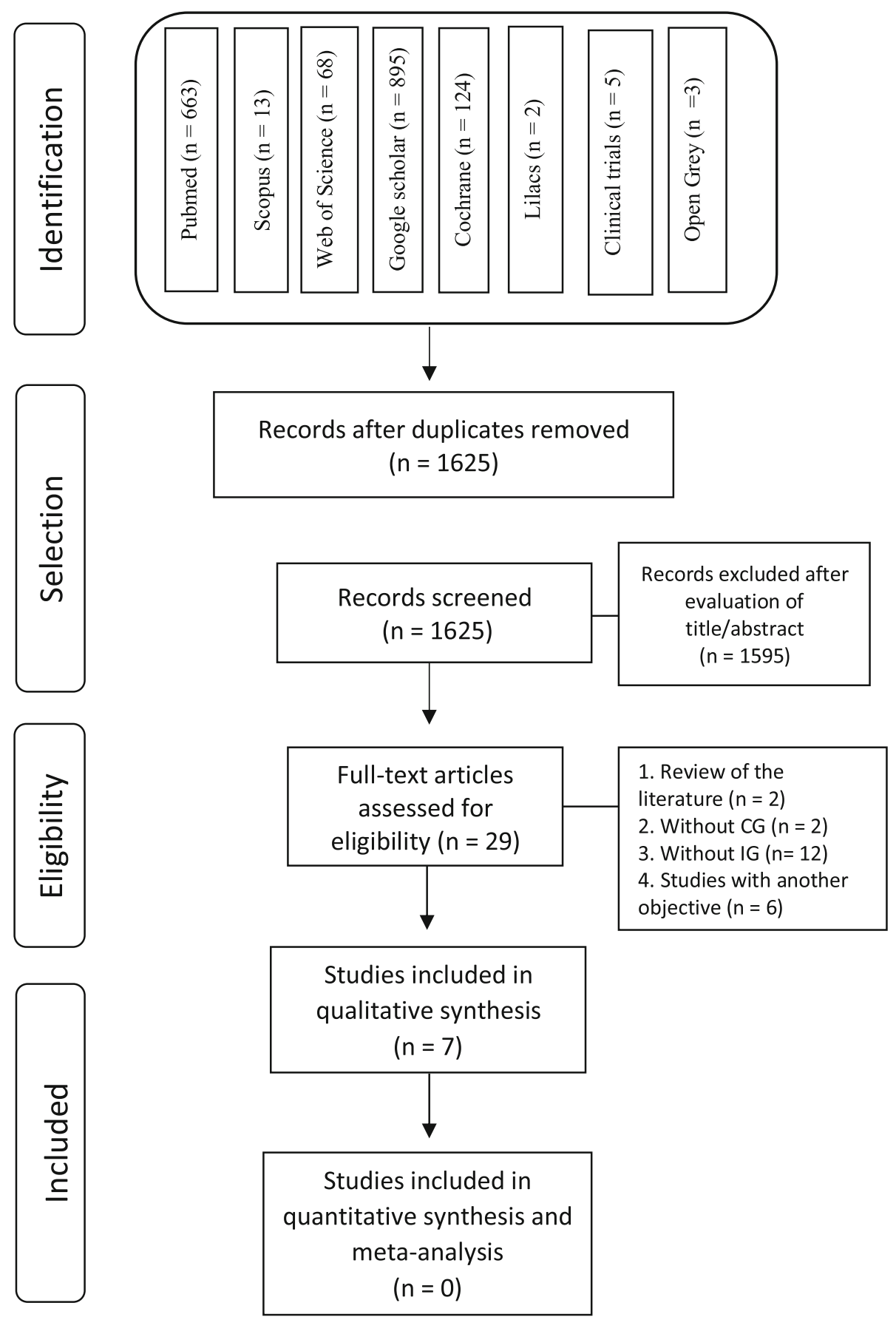

Fig. 1 Diagram with number of records at each stage of the review according to PRISMA statement

differences $(p>0.05)$ in pain level between groups. Only one study [2] performed this evaluation 2 months after starting treatment, and significant differences were found only on day $1(p=0.045)$ and day $2(p=0.041)$, with higher levels of pain in the control groups.

One study [25] compared different prescriptions of self-ligating appliances, Speed vs Damon, with Invisalign. Statistical differences were found between the
Speed and Invisalign prescription only in the first activation, .016" NiTi versus first aligner, and in the fourth phase, .019" $\times .025^{\prime \prime} \mathrm{CuNiTi}$ and tenth aligner, 3 days after a follow-up appliance. In these two evaluations, the group that used a fixed appliance presented higher levels of pain when compared to the Invisalign group. Although one paper [12] reported a higher pain level for the aligner group for all 
Table 5 List of excluded studies (with reason)

\begin{tabular}{ll}
\hline Reference & Reason for exclusion \\
\hline Abu Alhaija et al. (2015) & No intervention group \\
Ashkenazi et al. (2014) & No intervention group \\
Bergius et al. (2000) & Literature review \\
Bretz et al. (2018) & No intervention group \\
Caniklioglu et al. (2004) & No intervention group \\
Djeu et al (2014) & No pain evaluation \\
Fetouh (2008) & No pain evaluation \\
Fleming et al. (2009) & No intervention group \\
Kavaliauskiene et al. (2012) & No intervention group \\
Ke et al. (2019) & No pain evaluation \\
Kim (2013) & No control group \\
Mai-Tam (2018) & Literature review \\
Maldotti et al. (2013) & No control group \\
Noll et al. (2017) & Study not related with the SR objective \\
Pacheco-Pereira et al. (2015) & Study not related with the SR objective \\
Phuong (2018) & Study not related with the SR objective \\
Polat (2007) & No intervention group \\
Rakhshan (2015) & No intervention group \\
Salcedo-Bugarín (2018) & No intervention group \\
Scheurer et al. (1996) & No intervention group \\
Sergl et al. (1998) & No intervention group \\
Zealaiy et al. (2018) & No intervention group \\
\hline SR systmati rvew & \\
\hline
\end{tabular}

$S R$ systematic review evaluation times, $24 \mathrm{~h}$ and 14 days, no statistically significant $(p>0.05)$ difference was found for any time point.

Five studies $[2,10-12,25]$ reported the use of analgesics, of which three studies found statistical differences in time points for $4 \mathrm{~h}[10](p=0.001), 24 \mathrm{~h}[10,25](p=$ 0.001 and $p=0.025)$, day $2[2,25](p=0.0023$ and $p<$ $0.05)$, and day $3[11](p=0.006)$, and in all these cases, patients treated with fixed appliances reported a higher analgesic consumption. Only one study [12] observed that analgesic use was higher in the Invisalign group, since they discontinued their use on day 6 , which was different from the control group that stopped their use on day 4. One study [13] also assessed QoL and patient satisfaction during orthodontic treatment, finding a statistical difference only in the evaluation of eating and chewing, where the Invisalign group presented a better response than the control group ( $47 \%$ and $24 \%$, respectively).

Soft tissue irritation was reported to be lower in the Invisalign group in two studies $[12,25]$ as well as the assessments related to eating disorders [12].

\section{Certainty level}

The quality of the articles was assessed using the GRADE system described in Table 7. All timepoints evaluated in the studies were rated with low certainty of evidence in all CCT studies [10-12, 20, 25], except for the RCT [2] that was rated with high certainty of evidence. Just one study [13] was not included in the evaluation because it was a crosssectional study and had not made timepoint evaluations.

\section{Synthesis of results and additional analyses}

It was not possible to perform a meta-analysis because of large amount of clinical, methodological, and statistical

Table 6 Risk of bias of the included studies, according to the ROBINS-I tool

\begin{tabular}{|c|c|c|c|c|c|c|c|c|}
\hline \multicolumn{9}{|l|}{ Domains } \\
\hline \multirow[b]{2}{*}{ Author } & \multicolumn{2}{|c|}{ Pre-intervention } & \multirow{2}{*}{$\begin{array}{l}\text { Intervention } \\
\text { Bias in } \\
\text { classifying } \\
\text { interventions }\end{array}$} & \multicolumn{4}{|l|}{ Post-intervention } & \multirow[b]{2}{*}{$\begin{array}{l}\text { Overall risk } \\
\text { of bias } \\
\text { judgment }\end{array}$} \\
\hline & $\begin{array}{l}\text { Bias due to } \\
\text { confounding }\end{array}$ & $\begin{array}{l}\text { Bias in selection of } \\
\text { participants for the } \\
\text { study }\end{array}$ & & $\begin{array}{l}\text { Bias due to deviations } \\
\text { from intended } \\
\text { interventions }\end{array}$ & $\begin{array}{l}\text { Bias due to } \\
\text { missing } \\
\text { data }\end{array}$ & $\begin{array}{l}\text { Bias to } \\
\text { measuring } \\
\text { outcomes }\end{array}$ & $\begin{array}{l}\text { Bias in } \\
\text { selecting } \\
\text { reported } \\
\text { results }\end{array}$ & \\
\hline $\begin{array}{l}\text { Almasoud } \\
(2018)\end{array}$ & Low & Low & Low & Moderate & Low & Low & Low & Moderate \\
\hline $\begin{array}{l}\text { Fujiyama } \\
\text { et al. } \\
(2014)\end{array}$ & Moderate & Low & Moderate & Low & Low & Low & Moderate & Moderate \\
\hline $\begin{array}{l}\text { Mais- } \\
\text { Damois } \\
\text { et al. } \\
\text { (2015) }\end{array}$ & Moderate & Low & Low & Moderate & Low & Low & Moderate & Moderate \\
\hline $\begin{array}{l}\text { Miller et al. } \\
(2007)\end{array}$ & Moderate & High & High & Moderate & Low & Low & Low & High \\
\hline $\begin{array}{l}\text { Shalish } \\
\text { et al. } \\
(2007)\end{array}$ & Moderate & Moderate & Moderate & Moderate & Low & Low & Low & Moderate \\
\hline
\end{tabular}




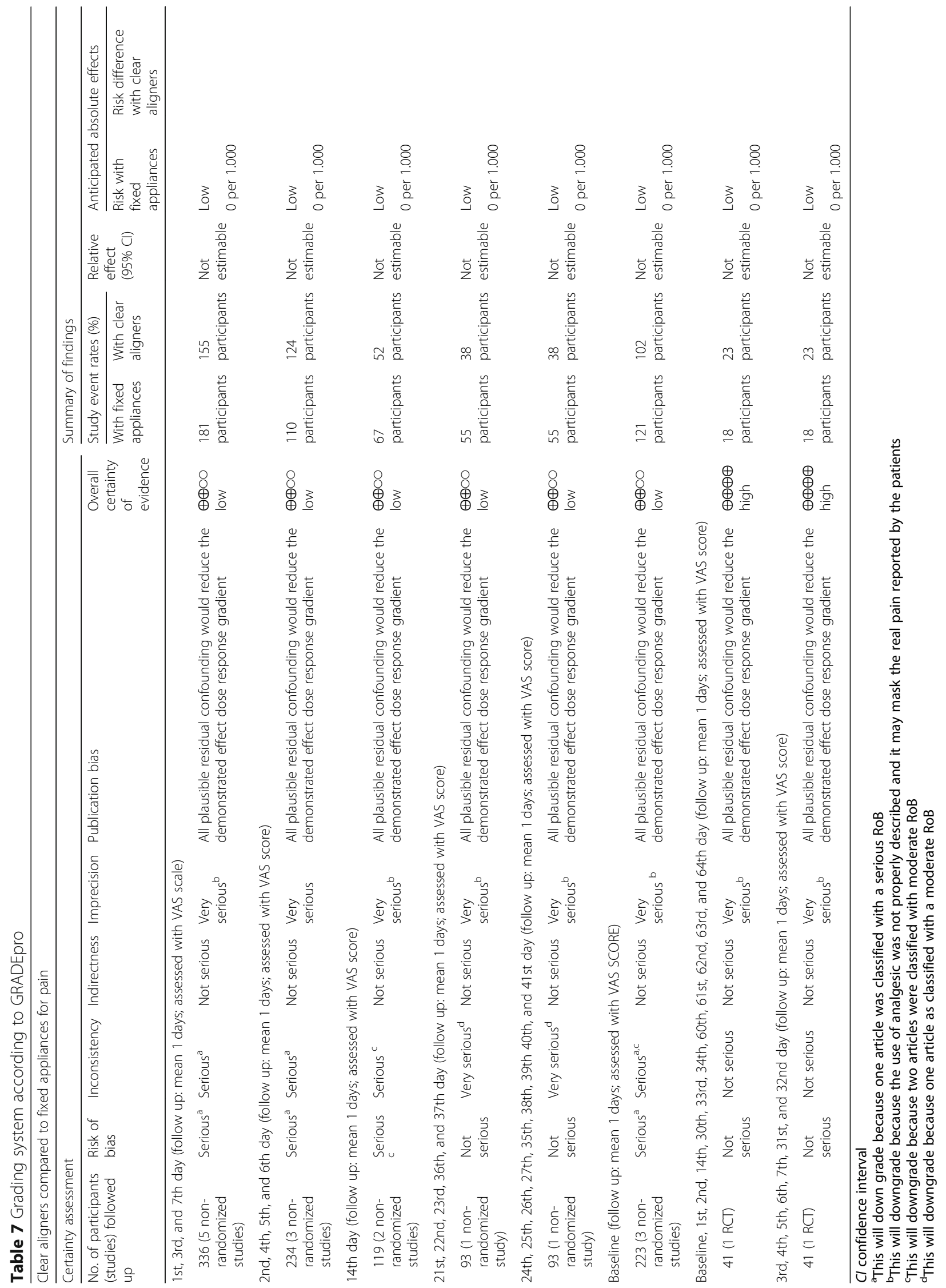


heterogeneity among the included studies, mainly due to differences between the archwire sequence in the fixed appliances and times of change for the aligners. In addition, attempts were made to contact the authors by email to collect missing data; however, only two of them responded, and they sent all the data available. Additional information still was not useful enough to justify a meta-analysis.

\section{Discussion}

In recent years, continuous search for esthetic alternatives and comfortable orthodontic treatment approaches have been reasons for significant increases in the number of cases treated with clear aligners. Recent studies have shown that patients specifically treated with Invisalign were satisfied with their esthetic results and showed an improvement in their QoL, especially when related to their smile and during chewing and eating functions analyzed after treatment $[13,27]$. However, concerning the efficacy of treatment, recent systematic reviews have suggested that this treatment modality presents some difficulties on specific orthodontic movements when compared with fixed appliances such as in rotation and vertical movements [14], ideal occlusal contacts, torque control, increasing transverse width and retention [16]. In addition, a study that evaluated the results of treatments performed with Invisalign and conventional brackets according to the American Board of Orthodontics' objective classification system showed that treatment with fixed appliances are relatively superior than the treatment performed with Invisalign [28].

Despite the fact that fixed appliances have been the most effective traditional method for orthodontic treatment for many years and have shown good treatment efficiency, several studies have reported the negative side effects of this technique, especially plaque accumulation and difficulty of oral hygiene [26, 29]. Another important aspect commonly observed is pain experience and discomfort during orthodontic treatment [30] since 91-95\% of patients experience some level of pain at different stages of treatment [8].

Pain is provoked by noxious stimuli and is a complex experience [30]. Therefore, it is important to understand the pain pattern during orthodontic treatment because pain and discomfort are two of the main reasons that affect the patient's QoL during treatment [31]. In addition, fear of pain is one of the main reasons for discouraging orthodontic treatment [32] and previous studies have found that $8 \%$ [33] to $30 \%$ [34] of patients discontinue orthodontic treatment due to pain experienced at the early stages of treatment.

Four studies [10, 11, 13, 25] reported higher levels of pain for the group treated with fixed appliances during the first $24 \mathrm{~h}$ after beginning treatment, which corroborates with other studies $[6,35,36]$, which show that the highest levels of pain are found 1 day after inserting initial archwires. Furthermore, the literature also shows that the pain is more intense during the first 3 days and is slowly minimized or disappears on the seventh day. This is in agreement with most of the included studies of this systematic review $[2,10,11,20]$. This pattern of pain occurs due to initial orthodontic forces that cause discomfort due to compression of the periodontal ligament, leading to ischemia, edema [37], and release of inflammatory mediators during the first 24-48 $\mathrm{h}$ [38]. These mediators such as prostaglandins (e.g., PgE) and interleukins (e.g., IL-1 $\beta$ ) sensitize nociceptors of the periodontal ligament, increasing discomfort. The levels of these mediators found in the gingival cervical fluid peak $24 \mathrm{~h}$ after the onset of orthodontic force and return to the reference values after 7 days [39]. This explains the pattern of pain observed during the first week after the application of orthodontic force.

Although the periods of higher and lower pain levels were similar for the fixed and Invisalign treated groups, in the present systematic review only one study [12] showed higher levels of pain for the group treated with aligners. They reported that this result may have been found due to a greater mechanical force applied at the beginning of Invisalign treatment; however, the sequence, time of use, and period of exchange of the aligners were not described.

Understanding that pain can affect the QoL of the individual, which can lead to worsening oral hygiene and have a psychosocial impact [40], many patients use analgesics for pain relief caused by orthodontic treatment. In the present systematic review, five studies reported the use of analgesics $[2,10-12,25]$, and in all of them, the use of analgesics was similar to the periods of higher and lower pain levels. The perception of orthodontic pain is due to changes in blood flow caused by the appliances, and the use of analgesic may reduce the inflammatory process, consequently reducing the pain levels [41], although the use of these pain relief medications may mask the real pain reported by the patients leading to an uncertain result. Medication intake was higher in the fixed appliance group than in the Invisalign group as previously reported in the literature $[8,35]$. The fact that patients with fixed appliances take more medications may underestimate the pain reported by them when treated with this type of appliance.

However, pain is a subjective process and can be influenced by several factors. Studies show that pain may be related to the individual's personality and that patients who have some knowledge about orthodontic treatment and have more positive attitudes presented lower levels of pain during treatment $[42,43]$. Therefore, it is suggested that the professionals inform the patients of any discomfort that may occur during orthodontic treatment and guide ways to alleviate it [42].

Knowing that the activation of the fixed appliance is done once a month and the aligners changed every 15 days, it may be reasonable to think that patients treated with aligners report lower pain levels at each activation, but it is felt for a longer period of time. That said, it is 
important to point out that few studies have evaluated pain over a longer period of treatment. A randomized clinical trial [2] performed this evaluation for 2 months and observed the pain in the subsequent appointments was lower in both groups. In the second month of maintenance, no statistical difference was observed.

The types of archwires should be taken in account since they have differences in some mechanical properties such as low elasticity module and coefficient of attrition, high resilience, flexibility and elastic recovery, and biocompatibility that are important characteristics to stimulate the adequate tissue response [44-46]. A laboratory study demonstrates that nickel-titanium archwire with addition of copper (CuNiTi) presented less favorable biologically deactivation loads compared to the other thermoactivated $\mathrm{NiTi}$ [47] which is consistent with a systematic review and meta-analyses [48] that found that patients treated with $\mathrm{CuNiTi}$ archwires presented greater levels of pain in the Likert scale than those patients treated with $\mathrm{NiTi}$.

However, lower levels of pain found in patients treated with Invisalign may be related to the fact that removable appliances produce less tension, pressure, sensitivity, and pain than fixed appliances [49]. This reduced discomfort in clear aligners may be associated with proinflammatory mediators such as IL- $1 \beta$ because in the short term, these mediators increase sensitization by triggering receptorassociated kinases and ion channels, and in the long term, they persuade the transcriptional upregulation of receptors, leading to hyperalgesia [50]. So, it is reasonable to state that removable appliances predisposed to painless responses due to the intermittent forces when compared to the continuous forces of the fixed appliances [51]. Furthermore, they can be removed by the patients themselves for pain relief. In addition, it was hypothesized by one study [12] that these results among non-randomized investigations should be evaluated with caution since cases treated with Invisalign usually have lower rates of irregularity index, and this difference may influence the patient's perception of pain, which is considered an important bias in the interpretation of the results. In this systematic review, only two studies [2, 10] considered crowding level as inclusion criteria, and in both of them, they range from mild to moderate. However, the other five studies [11-13,20,25] did not describe any information, and none of the included studies reported any differences in irregularity index between the evaluated groups. Despite that, there are controversial results about the correlation between the irregularity index and the perception of pain. Some studies found that there is no correlation [52-54], but a recent one found that crowding is a risk factor, and with each increase in crowding, there is a 1.10 times increase in painful sensation [55].

Another relevant factor is the type of malocclusion included in the studies. Some studies did not report inclusion criteria adequately $[11-13,20]$, and among those who reported [2, 10, 25], all included patients with mild or moderate malocclusion, Angle Class I malocclusion, and crowding of up to $5 \mathrm{~mm}$, which may bias the results, since the more severe the malocclusion, the more it is related to the psychosocial well-being of the patient in pain-related scales, psychological discomfort, and social problems [56].

Overall, the present systematic review showed lower pain levels for the groups treated with Invisalign during the first days of treatment. The studies presented a high methodological quality according to the grading system, with the RoB varying from moderate $[12,20,25]$ to high in five studies $[11,13]$, and only two $[2,10]$ of the studies presented a low RoB. Pain is one of many considerations, and predictability and technical outcome are more important, mainly considering that the difference does not seem to occur after the first months of the orthodontic treatment.

\section{Limitations}

There is a high level of heterogeneity in the design of the studies included in this systematic review. Among these studies, we observed a great variation in relation to the types of fixed appliance used, and five different types were externally funded by companies. In addition, the sequence of the archwires used and the set of the aligner was poorly detailed. Both factors can strongly affect the results found in this systematic review.

Selection of the participants was only randomized in one study [2] that presented a high certainty of evidence. In all other studies [10-12, 20, 25], that were classified with low certainty of evidence, selection was done according to the order of appearance of patients seeking orthodontic treatment, and in some cases, the patient chose which type of device they wanted to be treated with.

In addition, the use of analgesics was not reported in all studies. This may be likely a significant confounding factor since it is well established in the literature that the use of this drug camouflages the actual levels of pain produced during orthodontic treatment.

No other clear aligner appliances were studied in the included studies. No conclusions/suggestions can therefore be made about other alternatives.

\section{Conclusion}

Orthodontic patients treated with Invisalign appear to report lower levels of pain than those treated with fixed appliances during the first few days of treatment. However, the type of malocclusions was not comprehensively described which may lead to controversial results. Thereafter (up to 3 months), differences were not noted. Malocclusion complexity level among included studies was mild.

Based on the level of certainty, the results should be evaluated with caution, and it is suggested that studies with better methodological qualities be performed. 


\section{Appendix}

Table 8 Database and Search Strategy

Database Search Strategy

Pubmed Search ((c(c((((Orthodontic Appliances[MeSH Terms]) OR Orthodontic Appliances[Title/Abstract]) OR Appliance, Orthodontic[Title/ Abstract]) AND Appliances, Orthodontic[Title/Abstract]) OR Orthodontic Appliance[Title/Abstract])) OR

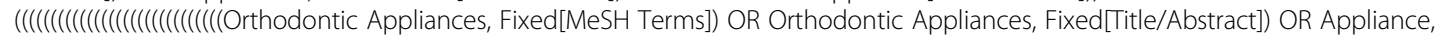
Fixed Orthodontic[Title/Abstract]) OR Appliances, Fixed Orthodontic[Title/Abstract]) OR Fixed Orthodontic Appliance[Title/ Abstract]) OR Fixed Orthodontic Appliances[Title/Abstract]) OR Orthodontic Appliance, Fixed[Title/Abstract]) OR Fixed Functional Appliances[Title/Abstract]) OR Appliance, Fixed Functional[Title/Abstract]) OR Appliances, Fixed Functional[Title/Abstract]) OR Fixed Functional Appliance[Title/Abstract]) OR Functional Appliance, Fixed[Title/Abstract]) OR Functional Appliances, Fixed[Title/ Abstract]) OR Fixed Retainer[Title/Abstract]) OR Fixed Retainers[Title/Abstract]) OR Retainer, Fixed[Title/Abstract]) OR Retainers, Fixed[Title/Abstract]) OR Bonded Retainer[Title/Abstract]) OR Bonded Retainers[Title/Abstract]) OR Retainer, Bonded[Title/Abstract]) OR Retainers, Bonded[Title/Abstract]) OR Fixed Appliances[Title/Abstract]) OR Appliance, Fixed[Title/Abstract]) OR Appliances, Fixed[Title/Abstract]) OR Fixed Appliance[Title/Abstract]) OR Permanent Retainer[Title/Abstract]) OR Permanent Retainers[Title/

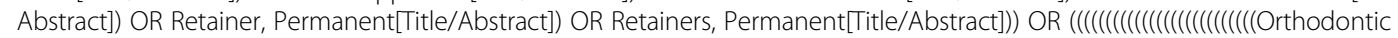
Appliances, Removable[MeSH Terms]) OR Orthodontic Appliances, Removable[Title/Abstract]) OR Appliance, Removable Orthodontic[Title/Abstract]) OR Appliances, Removable Orthodontic[Title/Abstract]) OR Orthodontic Appliance, Removable[Title/ Abstract]) OR Removable Orthodontic Appliance[Title/Abstract]) OR Removable Orthodontic Appliances[Title/Abstract]) OR Clear Dental Braces[Title/Abstract]) OR Brace, Clear Dental[Title/Abstract]) OR Braces, Clear Dental[Title/Abstract]) OR Clear Dental Brace[Title/Abstract]) OR Dental Brace, Clear[Title/Abstract]) OR Dental Braces, Clear[Title/Abstract]) OR Clear Aligner Appliances[Title/Abstract]) OR Aligner Appliance, Clear[Title/Abstract]) OR Aligner Appliances, Clear[Title/Abstract]) OR Appliance, Clear Aligner[Title/Abstract]) OR Appliances, Clear Aligner[Title/Abstract]) OR Clean Dental Braces[Title/Abstract]) OR Brace, Clean Dental[Title/Abstract]) OR Braces, Clean Dental[Title/Abstract]) OR Clean Dental Brace[Title/Abstract]) OR Dental Brace, Clean[Title/ Abstract]) OR Dental Braces, Clean[Title/Abstract]) OR Invisalign[Title/Abstract]) OR Invisaligns[Title/Abstract])) OR (((Traditional Brackets[Title/Abstract]) OR Edgewise appliance[Title/Abstract]) OR Passive self-ligating[Title/Abstract]))) AND ((((c(((Pain Perception[MeSH Terms]) OR Pain Perception[Title/Abstract]) OR Pain Perceptions[Title/Abstract]) OR Perception, Pain[Title/

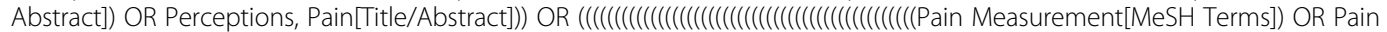
Measurement[Title/Abstract]) OR Measurement, Pain[Title/Abstract]) OR Measurements, Pain[Title/Abstract]) OR Pain Measurements[Title/Abstract]) OR Assessment, Pain[Title/Abstract]) OR Assessments, Pain[Title/Abstract]) OR Pain Assessments[Title/Abstract]) OR Pain Assessment[Title/Abstract]) OR Analgesia Tests[Title/Abstract]) OR Analgesia Test[Title/ Abstract]) OR Test, Analgesia[Title/Abstract]) OR Tests, Analgesia[Title/Abstract]) OR Nociception Tests[Title/Abstract]) OR Nociception Test[itile/Abstract]) OR Test, Nociception[Title/Abstract]) OR Tests, Nociception[Title/Abstract]) OR McGill Pain Questionnaire[Title/Abstract]) OR Pain Questionnaire, McGill[Title/Abstract]) OR Questionnaire, McGill Pain[Title/Abstract]) OR McGill Pain Scale[Title/Abstract]) OR Pain Scale, McGill[Title/Abstract]) OR Scale, McGill Pain[Title/Abstract]) OR Visual Analog Pain Scale[Title/Abstract]) OR Visual Analogue Pain Scale[Title/Abstract]) OR Analogue Pain Scale[Title/Abstract]) OR Analogue Pain Scales[Title/Abstract]) OR Pain Scale, Analogue[Title/Abstract]) OR Pain Scales, Analogue[Title/Abstract]) OR Scale, Analogue Pain[Title/Abstract]) OR Scales, Analogue Pain[Title/Abstract]) OR Analog Pain Scale[Title/Abstract]) OR Analog Pain Scales[Title/ Abstract]) OR Pain Scale, Analog[ititle/Abstract]) OR Pain Scales, Analog[itile/Abstract]) OR Scale, Analog Pain[Title/Abstract]) OR Scales, Analog Pain[Title/Abstract]) OR Formalin Test[Title/Abstract]) OR Formalin Tests[Title/Abstract]) OR Test, Formalin[Title/ Abstract]) OR Tests, Formalin[Title/Abstract]) OR Tourniquet Pain Test[Title/Abstract]) OR Pain Test, Tourniquet[Title/Abstract]) OR Pain Tests, Tourniquet[Title/Abstract]) OR Test, Tourniquet Pain[Title/Abstract]) OR Tests, Tourniquet Pain[Title/Abstract]) OR Tourniquet Pain Tests[Title/Abstract])) OR (((((Patient Comfort[MeSH Terms]) OR Patient Comfort[Title/Abstract]) OR Comfort, Patient[Title/Abstract]) OR Comfort Care[Title/Abstract]) OR Care, Comfort[Title/Abstract])) OR (((((Toothache[MeSH Terms]) OR Toothache[Title/Abstract]) OR Toothaches[Title/Abstract]) OR Odontalgia[Title/Abstract]) OR Odontalgias[Title/Abstract])) Sort by: Best Match

Search (((()((((Orthodontic Appliances[MeSH Terms]) OR Orthodontic Appliances[Title/Abstract]) OR Appliance, Orthodontic[Title/ Abstract]) AND Appliances, Orthodontic[Title/Abstract]) OR Orthodontic Appliance[Title/Abstract])) OR

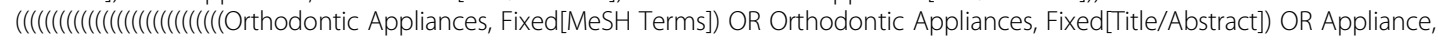
Fixed Orthodontic[Title/Abstract]) OR Appliances, Fixed Orthodontic[Title/Abstract]) OR Fixed Orthodontic Appliance[Title/ Abstract]) OR Fixed Orthodontic Appliances[Title/Abstract]) OR Orthodontic Appliance, Fixed[Title/Abstract]) OR Fixed Functional Appliances[Title/Abstract]) OR Appliance, Fixed Functional[Title/Abstract]) OR Appliances, Fixed Functional[Title/Abstract]) OR Fixed Functional Appliance[Title/Abstract]) OR Functional Appliance, Fixed[Title/Abstract]) OR Functional Appliances, Fixed[Title/ Abstract]) OR Fixed Retainer[Title/Abstract]) OR Fixed Retainers[Title/Abstract]) OR Retainer, Fixed[Title/Abstract]) OR Retainers, Fixed[Title/Abstract]) OR Bonded Retainer[Title/Abstract]) OR Bonded Retainers[Title/Abstract]) OR Retainer, Bonded[Title/Abstract]) OR Retainers, Bonded[Title/Abstract]) OR Fixed Appliances[Title/Abstract]) OR Appliance, Fixed[Title/Abstract]) OR Appliances, Fixed[Title/Abstract]) OR Fixed Appliance[Title/Abstract]) OR Permanent Retainer[Title/Abstract]) OR Permanent Retainers[Title/

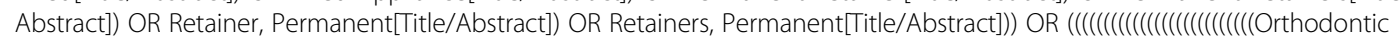
Appliances, Removable[MeSH Terms]) OR Orthodontic Appliances, Removable[Title/Abstract]) OR Appliance, Removable Orthodontic[Title/Abstract]) OR Appliances, Removable Orthodontic[Title/Abstract]) OR Orthodontic Appliance, Removable[Title/ Abstract]) OR Removable Orthodontic Appliance[Title/Abstract]) OR Removable Orthodontic Appliances[Title/Abstract]) OR Clear Dental Braces[Title/Abstract]) OR Brace, Clear Dental[Title/Abstract]) OR Braces, Clear Dental[Title/Abstract]) OR Clear Dental Brace[Title/Abstract]) OR Dental Brace, Clear[Title/Abstract]) OR Dental Braces, Clear[Title/Abstract]) OR Clear Aligner Appliances[Title/Abstract]) OR Aligner Appliance, Clear[Title/Abstract]) OR Aligner Appliances, Clear[Title/Abstract]) OR Appliance, Clear Aligner[Title/Abstract]) OR Appliances, Clear Aligner[Title/Abstract]) OR Clean Dental Braces[Title/Abstract]) OR Brace, Clean Dental[Title/Abstract]) OR Braces, Clean Dental[Title/Abstract]) OR Clean Dental Brace[Title/Abstract]) OR Dental Brace, Clean[Title/ Abstract]) OR Dental Braces, Clean[Title/Abstract]) OR Invisalign[Title/Abstract]) OR Invisaligns[Title/Abstract])) OR (((Traditional Brackets[Title/Abstract]) OR Edgewise appliance[Title/Abstract]) OR Passive self-ligating[Title/Abstract]))) AND ((((c(((Pain 
Table 8 Database and Search Strategy (Continued)

Database Search Strategy

Perception[MeSH Terms]) OR Pain Perception[Title/Abstract]) OR Pain Perceptions[Title/Abstract]) OR Perception, Pain[Title/

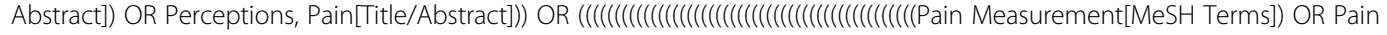
Measurement[Title/Abstract]) OR Measurement, Pain[Title/Abstract]) OR Measurements, Pain[Title/Abstract]) OR Pain Measurements[Title/Abstract]) OR Assessment, Pain[Title/Abstract]) OR Assessments, Pain[Title/Abstract]) OR Pain Assessments[Title/Abstract]) OR Pain Assessment[Title/Abstract]) OR Analgesia Tests[Title/Abstract]) OR Analgesia Test[Title/ Abstract]) OR Test, Analgesia[Title/Abstract]) OR Tests, Analgesia[Title/Abstract]) OR Nociception Tests[Title/Abstract]) OR Nociception Test[Title/Abstract]) OR Test, Nociception[Title/Abstract]) OR Tests, Nociception[Title/Abstract]) OR McGill Pain Questionnaire[Title/Abstract]) OR Pain Questionnaire, McGill[Title/Abstract]) OR Questionnaire, McGill Pain[Title/Abstract]) OR McGill Pain Scale[Title/Abstract]) OR Pain Scale, McGill[Title/Abstract]) OR Scale, McGill Pain[Title/Abstract]) OR Visual Analog Pain Scale[Title/Abstract]) OR Visual Analogue Pain Scale[Title/Abstract]) OR Analogue Pain Scale[Title/Abstract]) OR Analogue Pain Scales[Title/Abstract]) OR Pain Scale, Analogue[Title/Abstract]) OR Pain Scales, Analogue[Title/Abstract]) OR Scale, Analogue Pain[Title/Abstract]) OR Scales, Analogue Pain[Title/Abstract]) OR Analog Pain Scale[Title/Abstract]) OR Analog Pain Scales[Title/ Abstract]) OR Pain Scale, Analog[Title/Abstract]) OR Pain Scales, Analog[Title/Abstract]) OR Scale, Analog Pain[Title/Abstract]) OR Scales, Analog Pain[Title/Abstract]) OR Formalin Test[Title/Abstract]) OR Formalin Tests[Title/Abstract]) OR Test, Formalin[Title/ Abstract]) OR Tests, Formalin[Title/Abstract]) OR Tourniquet Pain Test[Title/Abstract]) OR Pain Test, Tourniquet[Title/Abstract]) OR Pain Tests, Tourniquet[Title/Abstract]) OR Test, Tourniquet Pain[Title/Abstract]) OR Tests, Tourniquet Pain[Title/Abstract]) OR Tourniquet Pain Tests[Title/Abstract])) OR ((((Patient Comfort[MeSH Terms]) OR Patient Comfort[Title/Abstract]) OR Comfort, Patient[Title/Abstract]) OR Comfort Care[Title/Abstract]) OR Care, Comfort[Title/Abstract])) OR (((((Toothache[MeSH Terms]) OR Toothache[Title/Abstract]) OR Toothaches[Title/Abstract]) OR Odontalgia[Title/Abstract]) OR Odontalgias[Title/Abstract]))

Final search: \#1 AND \#2

Scopus

( ( ( TITLE-ABS-KEY ( orthodontic AND appliances* ) OR TITLE-ABS-KEY ( "Appliance, Orthodontic" ) OR TITLE-ABS-KEY ( "Appliances, Orthodontic" ) OR TITLE-ABS-KEY ( "Orthodontic Appliance" ) ) ) OR ( ( TITLE-ABS-KEY ( orthodontic AND appliances, AND fixed* ) OR TITLE-ABS-KEY ( "Appliance, Fixed Orthodontic" ) OR TITLE-ABS-KEY ( "Appliances, Fixed Orthodontic" ) OR TITLE-ABS-KEY ( "Fixed Orthodontic Appliance" ) OR TITLE-ABS-KEY ( "Fixed Orthodontic Appliances" ) OR TITLE-ABS-KEY ( "Orthodontic Appliance, Fixed" ) OR TITLE-ABS-KEY ( "Fixed Functional Appliances" ) OR TITLE-ABS-KEY ( "Appliance, Fixed Functional" ) OR TITLE-ABS-KEY ( "Appliances, Fixed Functional" ) OR TITLE-ABS-KEY ( "Fixed Functional Appliance" ) OR TITLE-ABS-KEY ( "Functional Appliance, Fixed" ) OR TITLE-ABS-KEY ( "Functional Appliances, Fixed" ) OR TITLE-ABS-KEY ( "Fixed Retainer" ) OR TITLE-ABS-KEY ( "Fixed Retainers" ) OR TITLE-ABS-KEY ( "Retainer, Fixed" ) OR TITLE-ABS-KEY ( "Retainers, Fixed" ) OR TITLE-ABS-KEY ( "Bonded Retainer" ) OR TITLE-ABS-KEY ( "Bonded Retainers" ) OR TITLE-ABS-KEY ( "Retainer, Bonded" ) OR TITLE-ABS-KEY ( "Retainers, Bonded" ) OR TITLE-ABS-KEY ( "Fixed Appliances" ) OR TITLE-ABS-KEY ( "Appliance, Fixed" ) OR TITLE-ABS-KEY ( "Appliances, Fixed" ) OR TITLEABS-KEY ( "Fixed Appliance" ) OR TITLE-ABS-KEY ( "Permanent Retainer" ) OR TITLE-ABS-KEY ( "Permanent Retainers" ) OR TITLEABS-KEY ( "Retainer, Permanent" ) OR TITLE-ABS-KEY ( "Retainers, Permanent" ) ) ) OR ( ( TITLE-ABS-KEY ( orthodontic AND appliances, AND removable* ) OR TITLE-ABS-KEY ( "Appliance, Removable Orthodontic" ) OR TITLE-ABS-KEY ( "Appliances, Removable Orthodontic" ) OR TITLE-ABS-KEY ( "Orthodontic Appliance, Removable" ) OR TITLE-ABS-KEY ( "Removable Orthodontic Appliance" ) OR TITLE-ABS-KEY ( "Removable Orthodontic Appliances" ) OR TITLE-ABS-KEY ( "Clear Dental Braces" ) OR TITLE-ABSKEY ( "Brace, Clear Dental" ) OR TITLE-ABS-KEY ( "Braces, Clear Dental" ) OR TITLE-ABS-KEY ( "Clear Dental Brace" ) OR TITLE-ABSKEY ( "Dental Brace, Clear" ) OR TITLE-ABS-KEY ( "Dental Braces, Clear" ) OR TITLE-ABS-KEY ( "Clear Aligner Appliances" ) OR TITLEABS-KEY ( "Aligner Appliance, Clear" ) OR TITLE-ABS-KEY ( "Aligner Appliances, Clear" ) OR TITLE-ABS-KEY ( "Appliance, Clear Aligner" ) OR TITLE-ABS-KEY ( "Appliances, Clear Aligner" ) OR TITLE-ABS-KEY ( "Clear Aligner Appliance" ) OR TITLE-ABS-KEY ( "Clean Dental Braces" ) OR TITLE-ABS-KEY ( "Brace, Clean Dental" ) OR TITLE-ABS-KEY ( "Braces, Clean Dental" ) OR TITLE-ABS-KEY ( "Clean Dental Brace" ) OR TITLE-ABS-KEY ( "Dental Brace, Clean" ) OR TITLE-ABS-KEY ( "Dental Braces, Clean" ) OR TITLE-ABS-KEY ( "Invisalign" ) OR TITLE-ABS-KEY ( "Invisaligns" ) ) ) ) AND ( TITLE-ABS-KEY ( "invisalign" ) ) AND ( TITLE-ABS-KEY ( "pain" ) )

Web of Science ( ( TITLE-ABS-KEY ( orthodontic AND appliances*) OR TITLE-ABS-KEY ( "Appliance, Orthodontic" ) OR TITLE-ABS-KEY ( "Appliances, Orthodontic" ) OR TITLE-ABS-KEY ( "Orthodontic Appliance" ) ) ) OR ( ( TITLE-ABS-KEY ( orthodontic AND appliances, AND fixed*) OR TITLE-ABS-KEY ( "Appliance, Fixed Orthodontic" ) OR TITLE-ABS-KEY ( "Appliances, Fixed Orthodontic" ) OR TITLE-ABS-KEY ( "Fixed Orthodontic Appliance" ) OR TITLE-ABS-KEY ( "Fixed Orthodontic Appliances" ) OR TITLE-ABS-KEY ( "Orthodontic Appliance, Fixed" ) OR TITLE-ABS-KEY ( "Fixed Functional Appliances" ) OR TITLE-ABS-KEY ( "Appliance, Fixed Functional" ) OR TITLE-ABS-KEY ( "Appliances, Fixed Functional" ) OR TITLE-ABS-KEY ( "Fixed Functional Appliance" ) OR TITLE-ABS-KEY ( "Functional Appliance, Fixed" ) OR TITLE-ABS-KEY ( "Functional Appliances, Fixed" ) OR TITLE-ABS-KEY ( "Fixed Retainer" ) OR TITLE-ABS-KEY ( "Fixed Retainers" ) OR TITLE-ABS-KEY ( "Retainer, Fixed" ) OR TITLE-ABS-KEY ( "Retainers, Fixed" ) OR TITLE-ABS-KEY ( "Bonded Retainer" ) OR TITLE-ABS-KEY ( "Bonded Retainers" ) OR TITLE-ABS-KEY ( "Retainer, Bonded" ) OR TITLE-ABS-KEY ( "Retainers, Bonded" ) OR TITLE-ABS-KEY ( "Fixed Appliances" ) OR TITLE-ABS-KEY ( "Appliance, Fixed" ) OR TITLE-ABS-KEY ( "Appliances, Fixed" ) OR TITLEABS-KEY ( "Fixed Appliance" ) OR TITLE-ABS-KEY ( "Permanent Retainer" ) OR TITLE-ABS-KEY ( "Permanent Retainers" ) OR TITLEABS-KEY ( "Retainer, Permanent" ) OR TITLE-ABS-KEY ( "Retainers, Permanent" ) ) ) OR ( ( TITLE-ABS-KEY ( orthodontic AND appliances, AND removable* ) OR TITLE-ABS-KEY ( "Appliance, Removable Orthodontic" ) OR TITLE-ABS-KEY ( "Appliances, Removable Orthodontic" ) OR TITLE-ABS-KEY ( "Orthodontic Appliance, Removable" )

The Cochrane Library
\#1 (Malocclusion\$):ti,ab,kw OR ("Malocclusions"):ti,ab,kw OR ("Tooth Crowding"):ti,ab,kw OR ("Crowding, Tooth"):ti,ab,kw OR ("Crowdings, Tooth"):ti,ab,kw (Word variations have been searched)

\#2 (Malocclusion\$):ti,ab,kw OR (“Crossbite”):ti,ab,kw OR (“Crossbites"):ti,ab,kw OR (“Cross Bite”):ti,ab,kw OR (“Bite, Cross"):ti,ab,kw (Word variations have been searched)

\#3 (Malocclusion\$):ti,ab,kw OR ("Bites, Cross"):ti,ab,kw OR ("Cross Bites"):ti,ab,kw OR ("Angle's Classification"):ti,ab,kw OR ("Angle Classification"):ti,ab,kw (Word variations have been searched)

\#4 (Malocclusion\$):ti,ab,kw OR ("Angles Classification"):ti,ab,kw OR ("Classification, Angle's"):ti,ab,kw (Word variations have been searched)

\#5 \#1 and \#2 and \#3 and \#4

\#6 (Adult\$):ti,ab,kw OR ("Adults"):ti,ab,kw (Word variations have been searched) 
Table 8 Database and Search Strategy (Continued)

Database Search Strategy

\#7 (Orthodontic Appliances\$):ti,ab,kw OR ("Appliance, Orthodontic"):ti,ab,kw OR ("Appliances, Orthodontic"):ti,ab,kw OR

("Orthodontic Appliance"):ti,ab,kw (Word variations have been searched)

\#8 (Orthodontic Appliances, Fixed\$):ti,ab,kw OR ("Appliance, Fixed Orthodontic"):ti,ab,kw OR ("Appliances, Fixed Orthodontic"):ti,ab, kw OR ("Fixed Orthodontic Appliance"):ti,ab,kw OR ("Fixed Orthodontic Appliances"):ti,ab,kw (Word variations have been searched) \#9 (Orthodontic Appliances, Fixed\$):ti,ab,kw OR ("Orthodontic Appliance, Fixed"):ti,ab,kw OR ("Fixed Functional Appliances"):ti,ab, kw OR ("Appliance, Fixed Functional"):ti,ab,kw OR ("Appliances, Fixed Functional"):ti,ab,kw (Word variations have been searched) \#10 (Orthodontic Appliances, Fixed\$):ti,ab,kw OR ("Fixed Functional Appliance"):ti,ab,kw OR ("Functional Appliance, Fixed"):ti,ab,kw OR ("Functional Appliances, Fixed"):ti,ab,kw OR ("Fixed Retainer"):ti,ab,kw (Word variations have been searched)

\#11 (Orthodontic Appliances, Fixed\$):ti,ab,kw OR ("Fixed Retainers"):ti,ab,kw OR ("Retainer, Fixed"):ti,ab,kw OR ("Retainers, Fixed"):ti, $a b, k w$ OR ("Bonded Retainer"):ti,ab,kw (Word variations have been searched)

\#12 (Orthodontic Appliances, Fixed\$):ti,ab,kw OR ("Bonded Retainers"):ti,ab,kw OR ("Retainer, Bonded"):ti,ab,kw OR ("Retainers, Bonded"):ti,ab,kw OR ("Fixed Appliances"):ti,ab,kw (Word variations have been searched)

\#13 (Orthodontic Appliances, Fixed\$):ti,ab,kw OR ("Appliance, Fixed"):ti,ab,kw OR ("Appliances, Fixed"):ti,ab,kw OR ("Fixed Appliance"):ti,ab,kw OR ("Permanent Retainer"):ti,ab,kw (Word variations have been searched)

\#14 (Orthodontic Appliances, Fixed\$):ti,ab,kw OR ("Permanent Retainers"):ti,ab,kw OR ("Retainer, Permanent"):ti,ab,kw OR

("Retainers, Permanent"):ti,ab,kw (Word variations have been searched)

\#15 \#8 \#9 \#10 \#11 \#12 \#13 \#14

\#16 (Orthodontic Appliances, Removable\$):ti,ab,kw OR ("Appliance, Removable Orthodontic"):ti,ab,kw OR ("Appliances, Removable Orthodontic"):ti,ab,kw OR ("Orthodontic Appliance, Removable"):ti,ab,kw OR ("Removable Orthodontic Appliance"):ti,ab,kw (Word variations have been searched)

\#17 (Orthodontic Appliances, Removable\$):ti,ab,kw OR ("Removable Orthodontic Appliances"):ti,ab,kw OR ("Clear Dental Braces"):ti, ab,kw OR ("Brace, Clear Dental"):ti,ab,kw OR ("Braces, Clear Dental"):ti,ab,kw (Word variations have been searched)

\#18 (Orthodontic Appliances, Removable\$):ti,ab,kw OR ("Clear Dental Brace"):ti,ab,kw OR ("Dental Brace, Clear"):ti,ab,kw OR ("Dental Braces, Clear"):ti,ab,kw OR ("Clear Aligner Appliances"):ti,ab,kw (Word variations have been searched)

\#19 (Orthodontic Appliances, Removable\$):ti,ab,kw OR ("Aligner Appliance, Clear"):ti,ab,kw OR ("Aligner Appliances, Clear"):ti,ab,kw OR ("Appliance, Clear Aligner"):ti,ab,kw OR ("Appliances, Clear Aligner"):ti,ab,kw (Word variations have been searched)

\#20 (Orthodontic Appliances, Removable\$):ti,ab,kw OR (“Clean Dental Brace"):ti,ab,kw OR ("Dental Brace, Clean”):ti,ab,kw OR ("Dental Braces, Clean"):ti,ab,kw OR ("Invisalign"):ti,ab,kw (Word variations have been searched)

\#21 (Orthodontic Appliances, Removable\$):ti,ab,kw OR ("Invisaligns"):ti,ab,kw (Word variations have been searched)

\#22 \#16 \#17 \#18 \#19 \#20 \#21

\#23 (Pain Perception\$):ti,ab,kw OR ("Pain Perceptions"):ti,ab,kw OR (“Perception, Pain"):ti,ab,kw OR ("Perceptions, Pain"):ti,ab,kw (Word variations have been searched)

\#24 (Pain Measurement\$):ti,ab,kw OR ("Measurement, Pain"):ti,ab,kw OR ("Measurements, Pain"):ti,ab,kw OR ("Pain Measurements"): ti,ab,kw OR ("Assessment, Pain"):ti,ab,kw (Word variations have been searched)

\#25 (Pain Measurement\$):ti,ab,kw OR ("Assessments, Pain"):ti,ab,kw OR ("Pain Assessments"):ti,ab,kw OR ("Pain Assessment"):ti,ab,kw OR ("Analgesia Tests"):ti,ab,kw (Word variations have been searched)

\#26 (Pain Measurement\$):ti,ab,kw OR ("Analgesia Test"):ti,ab,kw OR (“Test, Analgesia"):ti,ab,kw OR ("Tests, Analgesia"):ti,ab,kw OR ("Nociception Tests"):ti,ab,kw (Word variations have been searched)

\#27 (Pain Measurement\$):ti,ab,kw OR ("Nociception Test"):ti,ab,kw OR (“Test, Nociception”):ti,ab,kw OR (“Tests, Nociception"):ti,ab, kw OR ("McGill Pain Questionnaire"):ti,ab,kw (Word variations have been searched)

\#28 (Pain Measurement\$):ti,ab,kw OR ("Pain Questionnaire, McGill"):ti,ab,kw OR ("Questionnaire, McGill Pain"):ti,ab,kw OR ("McGill Pain Scale"):ti,ab,kw OR ("Pain Scale, McGill"):ti,ab,kw (Word variations have been searched)

\#29 (Pain Measurement\$):ti,ab,kw OR ("Scale, McGill Pain"):ti,ab,kw OR ("Visual Analog Pain Scale"):ti,ab,kw OR ("Visual Analogue Pain Scale"):ti,ab,kw OR ("Analogue Pain Scale"):ti,ab,kw (Word variations have been searched)

\#30 (Pain Measurement\$):ti,ab,kw OR ("Analogue Pain Scales"):ti,ab,kw OR ("Pain Scale, Analogue"):ti,ab,kw OR ("Pain Scales, Analogue"):ti,ab,kw OR ("Scale, Analogue Pain"):ti,ab,kw (Word variations have been searched)

\#31 (Pain Measurement\$):ti,ab,kw OR ("Scales, Analogue Pain"):ti,ab,kw OR (“Analog Pain Scale"):ti,ab,kw OR ("Analog Pain Scales"): ti,ab,kw OR ("Pain Scale, Analog"):ti,ab,kw (Word variations have been searched)

\#32 (Pain Measurement\$):ti,ab,kw OR ("Pain Scales, Analog"):ti,ab,kw OR ("Scale, Analog Pain"):ti,ab,kw OR ("Scales, Analog Pain"):ti, ab,kw OR ("Formalin Test"):ti,ab,kw (Word variations have been searched)

\#33 (Pain Measurement\$):ti,ab,kw OR (“Formalin Tests"):ti,ab,kw OR (“Test, Formalin"):ti,ab,kw OR (“Tests, Formalin”):ti,ab,kw OR ("Tourniquet Pain Test"):ti,ab,kw (Word variations have been searched)

\#34 (Pain Measurement\$):ti,ab,kw OR ("Tourniquet Pain Tests"):ti,ab,kw (Word variations have been searched)

\#35 (Pain Measurement\$):ti,ab,kw OR ("Pain Test, Tourniquet"):ti,ab,kw OR ("Pain Tests, Tourniquet"):ti,ab,kw OR ("Test, Tourniquet

Pain"):ti,ab,kw OR ("Tests, Tourniquet Pain"):ti,ab,kw (Word variations have been searched)

\#36 \#24 \#25 \#26 \#27 \#28 \#29 \#30 \#31 \#32 \#33 \#34 \#35

\#37 (Patient Comfort\$):ti,ab,kw OR ("Comfort, Patient"):ti,ab,kw OR (“Comfort Care”):ti,ab,kw OR (“Care, Comfort”):ti,ab,kw (Word variations have been searched)

\#38 (Toothache\$):ti,ab, kw OR (“Toothaches"):ti,ab,kw OR (“Odontalgia”):ti,ab,kw OR (“Odontalgias"):ti,ab,kw (Word variations have been searched)

\#39 \#5 OR \#6

\#40 ("Traditional Brackets") OR ("Edgewise appliance") OR ("Passive self-ligating") (Word variations have been searched)

\#41 \#7 OR \#15 OR \#22 OR \#40

\#42 \#23 OR \#36 OR \# 37 OR \#38

\#43 \#39 AND \#41 AND \#42

\#44 \#30 AND \#41

\#45 \#39 AND \#42

\#46 \#41 AND \#42 
Table 8 Database and Search Strategy (Continued)

\begin{tabular}{ll}
\hline Database & Search Strategy \\
\hline & \#47 \#6 AND \#41 AND \#42 \\
& \#48 \#15 OR \#22 OR \#40 \\
& \#49 \#39 AND \#48 AND 42 \\
& \#50 \#48 AND \#42 \\
& \#51 "Invisalign" \\
& \#52 \#50 AND \#51 \\
& \#53 \#48 OR \#51 \\
& \#54 \#42 AND \#53 \\
& \#55 \#23 OR \#36 \\
& \#53 "discomfort" \\
& \#58 \#42 OR \#57 \\
& \#59 \#53 AND \#58 \\
& Invisalign AND pain \\
Lilacs & Invisalign \\
Open Grey & Adult + malocclusion + Orthodontic Appliances, Fixed + Orthodontic Appliances, Removable + comparison + Pain Measurement \\
Google Scholar & + Discomfort \\
Clinical Trials & Invisalign \\
OpenGrey & Invisalign
\end{tabular}

\section{Abbreviations}

CCT: Non-randomized clinical trial; GRADE: The Grading of Recommendations Assessment, Development and Evaluation; PRISMA: Preferred Reporting Items for Systematic Review and Meta-Analysis; QoL: Quality of life;

RCT: Randomized clinical trial; RoB: Risk of bias; ROBINS-I-Tool: Risk of Bias in Non-randomized Studies-of Interventions; VAS: Visual analog scale

\section{Acknowledgements}

Not applicable

\section{Authors' contributions}

PC realized the research and was the major contributor in writing the manuscript. DGE also realized the research and helped with the writing and with the all the stages of this article. PM helped with the research and corrected the writing. CFM corrected the writing and made and contributed with the correct structure of this article. DN corrected all the steps of this systematic review and corrected the writing. All authors read and approved the final manuscript.

\section{Funding}

Not applicable

\section{Availability of data and materials}

The datasets used and/or analyzed during the current study are available from the corresponding author on reasonable request.

\section{Ethics approval and consent to participate}

Not applicable

\section{Consent for publication}

Not applicable

\section{Competing interests}

The authors declare that they have no competing interests.

\section{Author details}

${ }^{1}$ Federal University of Pará (UFPA), Belém, Pará, Brazil. 'Faculty of Medicine and Dentistry, University of Alberta, Edmonton, Canada. ${ }^{3}$ Faculty of Dentistry, Federal University of Pará (UFPA), Rua Augusto Correa 01, Belém, Pará 66075-110, Brazil.
Received: 19 September 2019 Accepted: 13 December 2019

Published online: 20 January 2020

\section{References}

1. Bergius M, Kiliaridis S, Berggren U. Pain in orthodontics a review and discussion of the literature. J Orofac Orthop. 2000;62(2):125-37.

2. White DW, Julien KC, Jacob H, Campbell PM, Buschang PH. Discomfort associated with Invisalign and traditional brackets: a randomized prospective trial. Angle Orthod. 2017;87(6):801-8.

3. Kazancı F, Aydoğan C, Alkan Ö. Patients' and parents' concerns and decisions about orthodontic treatment. Korean J Orthod. 2016;46(1):20-6.

4. Al-Omiri MK, Abu Alhaija ES. Factors affecting patient satisfaction after orthodontic treatment. Angle Orthod. 2006;76(3):422-31.

5. Haynes S. Discontinuation of orthodontic relative to patient age Orthodontic. J Dent. 1967;2(4):138-42.

6. Jones M, Chan C. The pain and discomfort experienced during orthodntic treatment: a randomized controlled clinical trial of two intial aligning arch wires. Am J Orthod Dentofacial Orthop. 1992;102(4):373-81.

7. Ngan P, Kess B, Wilson S. Perception of discomfort by patients undergoing orthodontic treatment. Am J Orthod Dentofacial Orthop. 1989;96(1):47-53.

8. Scheurer PA, Firestone AR, Burgin WB. Perception of pain as a result of orthodontic treatment with fixed appliances. Eur J Orthod. 1996;18(4):349-57.

9. Thilander $B$, Rygh $P$, Reitan K. Tissue reactions in orthodontics.: Elsevier, Philadelphia, PA; 2005. 247-286 p.

10. Almasoud NN. Pain perception among patients treated with passive selfligating fixed appliances and Invisalign ${ }^{\circledR}$ aligners during the first week of orthodontic treatment. Korean J Orthod. 2018;48(5):326-32.

11. Miller KB, McGorray SP, Womack R, Quintero C, Perelmuter M, Gibson J, et al. A comparison of treatment impacts between Invisalign aligner and fixed appliance therapy during the first week of treatment. Am J Orthod Dentofacial Orthop. 2007;131(3):302.e1-9.

12. Shalish M, Cooper-kazaz R, Ivgi I, Canetti L, Tsur B, Bachar E, et al. Adult patients' adjustability to orthodontic appliances. Part I : a comparison

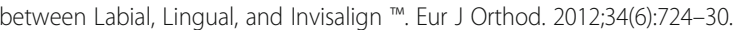

13. Flores-Mir C, Brandelli J, Pacheco-Pereira C. Patient satisfaction and quality of life status after 2 treatment modalities: Invisalign and conventional fixed appliances. Am J Orthod Dentofacial Orthop. 2018;154(5):639-44.

14. Galan-lopez L. A systematic review of the accuracy and efficiency of dental movements with Invisalign. Korean J Orthod. 2019;49(3):140-9.

15. Papadimitriou A, Mousoulea S, Gkantidis N, Kloukos D. Clinical effectiveness of Invisalign ${ }^{\oplus}$ orthodontic treatment: a systematic review. Prog Orthod. 2018;19(1):37.

16. Ke $Y$, Zhu $Y$, Zhu M. A comparison of treatment effectiveness between clear aligner and fixed appliance therapies. BMC Oral Health. 2019;19(1):24. 
17. Zheng M, Liu R, Ni Z, Yu Z. Efficiency, effectiveness and treatment stability of clear aligners: a systematic review and meta-analysis. Orthodo Craniofacial Res. 2017;20(3):127-33.

18. Rossini G, Parrini S, Castroflorio T, Deregibus A, Debernardi CL. Periodontal health during clear aligners treatment: a systematic review. Eur J Orthod. 2015;37(5):539-43.

19. Azaripour A, Weusmann J, Mahmoodi B, Peppas D, Gerhold-Ay A, Van Noorden CJF, et al. Braces versus Invisalign ${ }^{\circledR}$ : gingival parameters and patients ' satisfaction during treatment : a cross-sectional study. BMC Oral Health. 2015;15(1):69

20. Fujiyama K, Honjo T, Suzuki M, Matsuoka S, Deguchi T. Analysis of pain level in cases treated with Invisalign aligner : comparison with fixed edgewise appliance therapy. Prog Orthod. 2014;15(1):64.

21. Wells GA, Shea B, O'Connell D, Peterson J, Welch V, Losos M, et al. The Newcastle-Ottawa Scale (NOS) for assessing the quality of nonrandomised studies in meta-analyses. 2014.

22. Sterne JAC, Hernán MA, Reeves BC, Savović J, Berkman ND, Viswanathan M, et al. ROBINS-I : a tool for assessing risk of bias in non-randomised studies of interventions. BMJ. 2016;355:i4919-i.

23. Higgins JPT, Sterne JACJS, Page MJ, Hróbjartsson A, Boutron I, Reeves B, et al. A revised tool for assessing risk of bias in randomized trials. Cochrane Database Syst Rev. 2016;10(suppl 1):29-31.

24. Balshem H, Helfand M, Schunemann HJ, Oxman AD, Kunz R, Brozek J, et al. GRADE guidelines: 3. Rating the quality of evidence. J Clin Epidemiol. 2011;64(4):401-6.

25. Masi-damois C. Comparaison de la perception de la douleur entre le traitement orthodontique avec Invisalign ${ }^{\oplus}$ et le traitement avec fils et boîtiers fixes; 2015

26. Tufekcia E, Lucchese A, Gherlone E. Prevalence of white-spot lesions before and during orthodontic treatment with fixed appliances. Eur J Orthod. 2013; 35(5):664-8.

27. Pacheco-Pereira C, Brandelli J, Flores-Mir C. Patient satisfaction and quality of life changes after Invisalign treatment. Am J Orthod Dentofacial Orthop. 2018;153(6):834-41.

28. Djeu G, Shelton C, Maganzini A. Outcome assessment of Invisalign and traditional orthodontic treatment compared with the American Board of Orthodontics objective grading system. Am J Orthod Dentofacial Orthop. 2005;128(3):292-8.

29. Zachrisson S, Zachrisson BU. Gingival condition associated with orthodontic treatment. Angle Orthod. 1972;42(1):26-34.

30. Bergius $M$, Berggren U, Kiliaridis S. Experience of pain during an orthodontic procedure. Eur J Oral Sci. 2002;110(2):92-8.

31. Krukemeyer AM, Arruda AO, Inglehart MR. Pain and orthodontic treatment patient experiences and provider assessments. Angle Orthod. 2009;79(6): $1175-81$.

32. Oliver RG, Knapman YM. Attitudes to orthodontic treatment. Br J Orthod 2019;12(4):179-88.

33. Patel V. Non-completion of active orthodontic treatment. Br J Orthod. 2019; 19(1):47-54

34. Lew KK. Attitudes and perceptions of adults towards orthodontic treatment in an Asian community. J Community Dent Oral Epidemiol. 1993;21(1):31-5.

35. Jones ML. An investigation into the initial discomfort caused by placement of an archwire. Eur J Orthod. 1984;6(1):48-54.

36. Erdinç AME, Dinçer B. Perception of pain during orthodontic treatment with fixed appliances. Eur J Orthod. 2004;26(1):79-85.

37. Furstman L, Bernick S. Clinical considerations of the periodontium. Am J Orthod Dentofacial Orthop. 1970;61(2):138-55.

38. Dray A. Inflammatory mediators of pain. Br J Anaesthesia. 1995;75(2):125-31.

39. Grieve WG III, Johnson GK, Moore RN, Reinhardt RA, Dubois LM. Levels in gingival crevicular fluid during human orthodontic tooth movement. Am J Orthod Dentofacial Orthop. 1994;105(4):369-74.

40. Oliveira CMD, Sheiham A. Orthodontic treatment and its impact on oral health-related quality of life in Brazilian adolescents. J Orthod. 2004;31(1):20-7.

41. Krishnan V. Orthodontic pain: from causes to management--a review. Eur J Orthod. 2007;29(2):170-9.

42. Doll GM, Zentner A, Klages U, Sergl HG. Relationship between patient discomfort, appliance acceptance and compliance in orthodontic therapy. J Orofacial Orthop. 2000;61(6):398-413.

43. Bos A, Hoogstraten J, Prahl-andersen B. Attitudes towards orthodontic treatment : a comparison of treated and untreated subjects. Eur J Orthod. 2005;27(2):148-54
44. Burstone CJ, Goldberg AJ. Beta titanium: a new orthodontic alloy. 1980; 77(2):121-32.

45. Kapila S, Sachdeva RJAJoO, Orthopedics D. Mechanical properties and clinical applications of orthodontic wires. 1989;96(2):100-9.

46. Evans TJW, Jones ML, Newcombe RGJAjoo, orthopedics d. Clinical comparison and performance perspective of three aligning arch wires. 1998 114(1):32-9.

47. Gravina MA, Brunharo IHVP, Canavarro C, Elias CN, Quintão CCAJDpjoo. Mechanical properties of NiTi and CUNiTi shape-memory wires used in orthodontic treatment. Part 1: stress-strain tests. 2013;18(4):35-42.

48. Papageorgiou S, Konstantinidis I, Papadopoulou K, Jäger A, Bourauel CJO, research C. A systematic review and meta-analysis of experimental clinical evidence on initial aligning archwires and archwire sequences. 2014;17(4): 197-215.

49. Sergl HG, Klages $U$, Zentner A. Pain and discomfort during orthodontic treatment: causative factors and effects on compliance. Am J Orthod Dentofacial Orthop. 1998;114(6):684-91.

50. Opree A, Kress M. Involvement of the proinflammatory cytokines tumor necrosis factor- $\mathrm{a}, \mathrm{IL}-1 \mathrm{~b}$, and $\mathrm{IL}-6$ but not IL-8 in the development of heat hyperalgesia: effects on heat-evoked calcitonin gene-related peptide release from rat skin. J Neurosci. 2000;20(16):6289-93.

51. Polat Ö. Pain and discomfort after orthodontic appointments. Semin Orthod. 2007;13(4):292-300.

52. Abdelrahman RS, Al-Nimri KS, Al Maaitah EF. Pain experience during initial alignment with three types of nickel-titanium archwires: a prospective clinical trial. Angle Orthod. 2015;85(6):1021-6.

53. Da Costa E, Blagitz M, Normando A. Impact of catastrophizing on pain during orthodontic treatment. Dental Press J Ortho. 2019.

54. Brown DF, Moerenhout RG. The pain experience and psychological adjustment to orthodontic treatment of preadolescents, adolescents, and adults. Am J Orthod Dentofacial Orthop. 1991;100(4):349-56.

55. Salgado K, Normando A, Brandão G. Factors associated with pain perception in the initial phase of orthodontic alignment: a cohort study:: Federal University of Pará; 2018.

56. Sun L. Relationship between the severity of malocclusion and oral health related quality of life : a systematic review and meta-analysis. Oral Health Prev Dent. 2017;15(6):503-18.

\section{Publisher's Note}

Springer Nature remains neutral with regard to jurisdictional claims in published maps and institutional affiliations.

\section{Submit your manuscript to a SpringerOpen ${ }^{\circ}$ journal and benefit from:}

- Convenient online submission

- Rigorous peer review

- Open access: articles freely available online

- High visibility within the field

- Retaining the copyright to your article

Submit your next manuscript at $>$ springeropen.com 